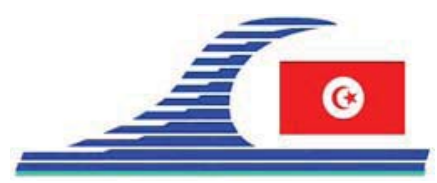

Conférence Méditerranéenne Côtière et Maritime EDITION 1, HAMMAMET, TUNISIE (2009)

Coastal and Maritime Mediterranean Conference

Disponible en ligne - http://www.paralia.fr-Available online

\title{
Master plan du nouveau port commercial de Shenjin, Albanie
}

\author{
Isuf BERBERI ${ }^{1}$, Lucia DE ANGELIS ${ }^{2}$, Marco GONELLA ${ }^{2}$, Paola LETIZIA ${ }^{3}$
}

1. OTTO-AL Group, Rr. "Elbasanit” Pall poshte Fak. Gjeologjise, Tirana, Albanie. isufberberi@gmail.com

2. MED Ingegneria S.r.1., Via Zangheri 16, 48100 Fornace Zarattini - Ravenne, Italie. lucia.deangelis@medingegneria.it; marco.gonella@medingegneria.it

3. MED Ingegneria S.r.1., Corso Stati Uniti 1/17, 35127 Padoue, Italie.

paola.letizia@medingegneria.it

\section{Résumé :}

Cette étude concerne la rédaction du Master Plan du nouveau port commercial de Shengjin, en Albanie.

Le but de cette étude de faisabilité est celui d'identifier la meilleure solution parmi plusieurs alternatives de développement du Port de Shengjin, en fonction des nécessités de l'industrie du ciment, et sur la base des futurs développements des transports routiers en Albanie et des trafics maritimes par rapport aux autres ports du Pays (Durres et Vlore), et dans la Mer Adriatique (corridor VIII), en essayant de créer un impact minimum sur l'environnement.

La planification portuaire du port de Shengjin a été définie en trois phases de développement dans lesquelles on prévoit des aménagements de défense côtière et des ouvrages portuaires, le dragage du chenal d'accès et du bassin de manœuvre et amarrage, des quais avec des installations de chargement/déchargement de navires, le mouvement des cargaisons, des grues conteneurs mobiles, dépôts et zones de stockage de marchandises, bandes transporteuses, silos verticaux pour ciment reliés aux quais par bandes transporteuses, tunnels pour la connexion avec l'hinterland.

En outre on a mené deux études spécifiques : une pour étudier les impacts des ouvrages sur l'environnement, et une étude météo-marine de la houle et analyse de l'hydrodynamique côtière et portuaire avec modélisation numérique.

Pour ces analyses on a utilisé la modélisation numérique bidimensionnelle, et on a appliqué en particulier le code de calcul MIKE21, mis au point par le Danish Hydraulic Institute (DHI, 2004).

\section{Mots-clés :}

Planification portuaire - Développement des trafics maritimes - Aménagements de défense côtière et ouvrages portuaires - Génie côtier - Impacts su l'environnement Hydrodynamique côtière et portuaire avec modélisation numérique

DOI: $10.5150 / \mathrm{cmcm} .2009 .003$ 


\section{Introduction}

L'objectif de l'étude de faisabilité et du projet est celui de développer et d'augmenter la capacité du port de Shengjin, situé dans le nord de l'Albanie, en modernisant les infrastructures actuelles et en optimisant l'efficacité des opérations portuaires de la façon suivante :

- Augmentation de la capacité, grâce à l'agrandissement des quais, des docks équipés pour le chargement et le déchargement des navires ;

- Optimisation des opérations portuaires, en améliorant l'efficacité et la capacité de gestion des chargements des cargaisons ;

- Rationalisation de l'utilisation des quais du terminal maritime;

- Agrandissement du réseau routier actuel ;

- Amélioration des qualités de toutes les opérations portuaires et des services effectués du point de vue rapport qualité-prix.

On a prévu le développement du port en trois phases :

1) Port au service de la cimenterie, avec une surface navigable de 19 hectares. Construction d'un tunnel de connexion $(3,1 \mathrm{~km})$.

2) Terminal «Ro-Ro» (Roll On - Roll off), avec une surface navigable de 53 hectares. Construction d'un deuxième tunnel $(3,2 \mathrm{~km})$.

3) Terminal conteneur (pour feeder), avec une surface navigable de 185 hectares. Construction d'un troisième tunnel $(3,2 \mathrm{~km})$.

\section{Méthodologie d'analyse}

L'étude de faisabilité pour le développement du port de Shengjin a été menée en plusieurs étapes.

La première concerne l'analyse de l'état actuel, à partir de l'origine et de l'histoire du port de Shengjin, ensuite les infrastructures actuelles du port, les aspects météo-marins (climat de la houle au large de Shengjin et sur le site à partir des données enregistrées extraites du « Wind and Waves Atlas of the Mediterranean Sea » publié en avril 2004 à la fin du Projet Europeéen MEDATLAS réalisé pendant les années 1999-2004), les impacts sur la géologie et sur l'environnement. On a effectué des levés bathymétriques, levés topographiques de la plage émergée, de la zone derrière le port, y compris la colline derrière le port, où devra passer le tunnel de connexion entre le port et l'hinterland, des levés des structures existantes et des analyses sédimentologiques. Les transformations morphologiques et extrêmes subies par les houles, pendant la propagation du large vers la côte à cause des phénomènes de shoaling, friction au fond, réfraction, diffraction et déferlement ont été étudiées en appliquant le modèle bidimensionnel MIKE21-PMS. Ensuite, le module MIKE21-HD a permis l'évaluation des courants provoqués par les houles. Pour l'analyse du transport des sédiments le long du littoral on a appliqué le module de calcul non-cohésif Sediment Transport MIKE21ST. A la base de la planification du nouveau port commercial il y a l'étude et l'analyse 
de : transports routiers, ferroviaires et maritimes en Albanie et dans la Mer Adriatique; le marché du ciment interne et en Europe; le contexte socio-économique du pays; les institutions, les plans, les réglementations et les aspects juridiques de l'Albanie. La seconde étape concerne l'évaluation des flux de trafic potentiels dans les ports de l'Albanie jusqu'en 2028, dans les secteurs du ciment et du charbon, des RO-RO («Roll On - Roll Off » et des containers (feeder).

Dans la troisième partie de l'étude on définit les objectifs de trafic et les critères pour les infrastructures maritimes, pour le tunnel et l'industrie du ciment. On propose deux alternatives possibles de projets pour le développement du port.

Dans la quatrième phase on définit le «master plan » du port de Shengjin, avec son développement en trois phases. On a développé le projet préliminaire de la première phase (port pour la cimenterie, avec quai pour dépôts de charbon) et l'étude de faisabilité du point de vue des impacts sur l'environnement; on a effectué une analyse détaillée économique et financière et l'analyse des impacts socio-économiques, pour définir la rentabilité de l'investissement.

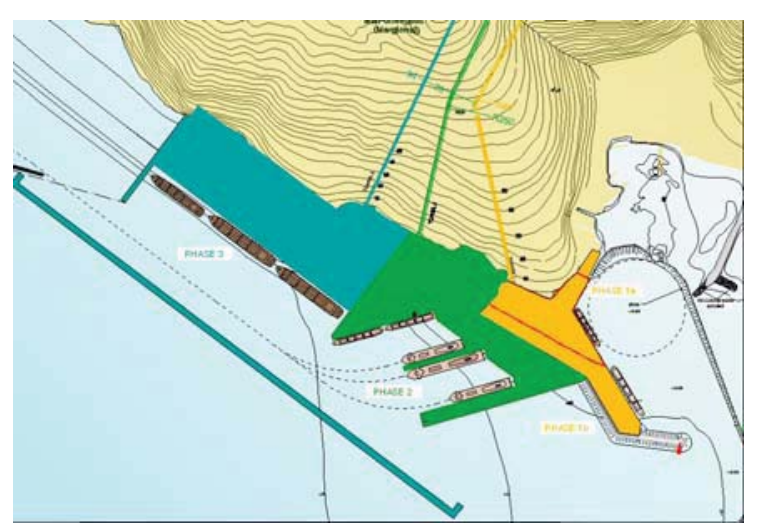

Figure 1. Développement du port de Shengjin, Albanie.

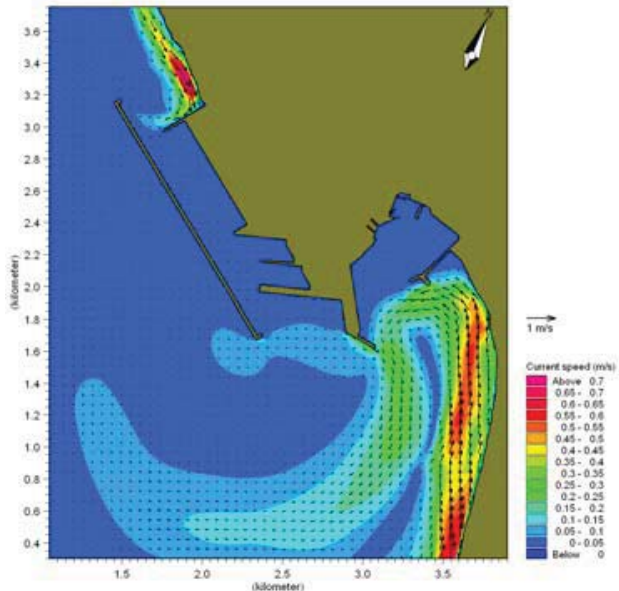

Figure 2. Champs des vitesses de courant calculés avec le module MIKE21-HD, configuration de projet - $3^{\text {ème }}$ phase.

\section{Résultats obtenus}

Cette étude a mis en évidence la nécessité d'agrandir le port de Shengjin en différentes phases pour pouvoir répondre aux prévisions de développement des trafics dans les prochaines 20 années (jusqu'en 2028).

Dans la première phase il est prévu de réaliser un port au service de l'industrie du ciment, qui se situe dans l'hinterland derrière la colline, et qui sera relié au port grâce au nouveau tunnel d'environ $3,1 \mathrm{~km}$. Les quais pourront desservir des navires jusqu'à 12000 DWT pour l'exportation du ciment et jusqu'à 15000 DWT pour l'importation du charbon. Les aménagements prévus pour rendre opérationnel le nouveau port sont les 
suivants : dragage du chenal d'accès actuel et du bassin de manœuvre et amarrage pour entretenir une profondeur suffisante $(10 \mathrm{~m})$; construction d'une nouvelle jetée à talus pour la protection des zone pour les installations terrestres, approfondissement de la jetée actuelle pour garantir une profondeur de $10 \mathrm{~m}$; reconstruction du quai actuel (100 m de longueur), construction d'une nouvelle darse de $200 \mathrm{~m}$ pour l'amarrage des navires de ciment et de charbon. Pour le chargement/déchargement du ciment et du charbon on prévoit des bandes transporteuses, 4 silos verticaux pour le ciment reliés aux quais par bandes transporteuses, une grue mobile sur rails. Pour le dépôt du charbon on prévoit un grand bassin.

La propagation de la houle, les courants le long du littoral et l'agitation de la houle à l'intérieur du bassin portuaire ont été étudiés en appliquant la modélisation numérique.

\section{Développements futurs}

Dès que le Master Plan sera approuvé par le Gouvernement et les Autorités locales, la solution retenue pour la première phase, devra être développée au niveau de projet détaillé.

La réalisation de la première phase est prévue en 3 ans, avec possibilité d'extension de la jetée et agrandissement des quais avec une nouvelle darse de $200 \mathrm{~m}$ (2 années de travaux supplémentaires); les phases 2 et 3 seront construites en 4 ans chacune. La fin des travaux (sans considérer l'extension de la $1^{\text {ère }}$ phase) est prévue en 2022.

\section{Bibliographie}

AIPCN/PIANC (2003). Breakwaters with vertical and inclined concrete walls, issued by the Permanent Association of navigation Congress.

DHI -Danish Hydraulic Institute- (2004). MIKE21, LIPACK Manuals, Horsholm, Denmark.

FAO (1999). Impact of the Kosovo Crisis on Albanian Agriculture and the Environment. Special Relief Operations Service - TCOR.

GODA Y. (1985). Random seas and design of maritime structures. University of Tokyo press, Tokyo, Japan.

GRIMALDI M. (2005). Corridor VIII development in the context of balkan region transport network: role and activities of corridor viii technical secretariat. 8th CEI Summit Economic Forum, November 2005, Bratislava, Slovak Republic.

INSTITUTE OF TRANSPORT STUDIES - REPUBBLIC OF ALBANIA, MINISTRY OF PUBLIC WORKS AND TRANSPORT (1999). Study on secondary ports: port of Shenjin. Tirana, Albanie.

MINISTRY OF TERRITORIAL ADJUSTEMENT AND TOURISM OF ALBANIA (2005). Albania Coastal Zone Development and Clean-up Program, World Bank project.

SIMEONI U., PANO N., CIAVOLA P. (1997). The coastline of Albany: morphology, evolution and coastal management issues. Bulletin de l'Institut Oceanographique, $\mathrm{N}^{\circ} 18$.

US DEPARTMENT OF THE ARMY (1984). Shore Protection Manual. Coastal Engineering Research Center, Washington, USA.

VAN DER MEER J.W. (1993). Conceptual design of rubble mound breakwaters, Delft Hydraulics, Delft, The Netherlands. 